\title{
Competence, specification and commitment in otic placode induction
}

\author{
Andrew K. Groves* and Marianne Bronner-Fraser \\ Division of Biology, 139-74, California Institute of Technology, Pasadena, CA 91125, USA \\ *Author for correspondence at present address: Department of Cell and Molecular Biology, House Ear Institute, 2100 West Third Street, Los Angeles, CA 90057, USA \\ (e-mail: agroves@hei.org)
}

Accepted 4 June; published on WWW 20 July 2000

\section{SUMMARY}

The inner ear is induced from cranial ectoderm adjacent to the hindbrain. Despite almost a century of study, the molecular mechanisms of inner ear induction remain obscure. We have identified four genes expressed very early in the anlage of the inner ear, the otic placode. Pax-2, Sox3, BMP-7 and Notch are all expressed in placodal ectoderm from the 4-5 somite stage (ss) onwards, well before the otic placode becomes morphologically visible at the 12-14ss. We have used these four molecular markers to show that cranial ectoderm becomes specified to form the otic placode at the 4-6ss, and that this ectoderm is committed to a placodal fate by the 10ss. We also demonstrate that much of the embryonic ectoderm is competent to generate an otic placode if taken at a sufficiently early age. We have mapped the location of otic placode-inducing activity along the rostrocaudal axis of the embryo, and have determined that this activity persists at least until the 10ss. Use of the four molecular otic placode markers suggests that induction of the otic placode in birds occurs earlier than previously thought, and proceeds in a series of steps that are independently regulated.

Key words: Otic Placode, Induction, Chicken, Competence, Specification, Commitment

\section{INTRODUCTION}

The concept of embryonic induction is central to an understanding of development. Embryonic induction has been defined as an interaction between an inducing and a responding tissue that alters the path of differentiation of the responding tissue (Gurdon, 1987; Jacobson, 1966; Jacobson and Sater, 1988). This phenomenon was first characterized by Spemann and Lewis at the turn of the century in their studies of lens induction (for reviews see Grainger, 1992, 1996), and since that time it has become clear that many, if not all tissues are formed as a result of inductive processes.

It is now accepted that embryonic induction rarely occurs in a single step, but rather as a series of changes in the responding tissue, leading ultimately to the complete differentiation of the tissue or organ (Gurdon, 1987; see Grainger, 1996 for a detailed account of lens differentiation; Ginsburg, 1995). Moreover, the steps comprising the induction of a tissue or cell type may be regulated independently from one another (e.g. Groves and Anderson, 1996; Groves et al., 1995). For these reasons it has become increasingly important to define molecular markers for each stage of a given inductive process to more fully understand the interactions between inducing and responding tissues.

The inner ear is an attractive system for the study of embryonic induction. It consists of several distinct classes of cells: sensory, non-sensory and neuronal, arranged in an organ of exquisitely complex morphology, and yet it is derived from a simple patch of placodal ectoderm, the otic placode, which arises next to the hindbrain (reviewed in Fritzsch et al., 1998;
Torres and Giráldez, 1998). Many studies over the last 80 years have examined the interactions between presumptive placodal ectoderm and candidate-inducing tissues leading to the formation of the inner ear. It is clear from these studies that much of the ectoderm in early chick and amphibian embryos is competent to generate an inner ear if grafted to an appropriate location, and that the competence of ectoderm outside the ear-forming region declines with age (e.g. Gallagher et al., 1996; Ginsburg, 1995; Jacobson, 1963a-c; Kaan, 1926; Yntema, 1950, 1955). Within the presumptive otic placode region, however, inductive signals act to specify and then commit the placodal ectoderm to an inner ear fate.

Studies on inner ear induction to date have almost always relied on the formation of an otic vesicle as an assay for successful induction. The appearance, however, of a morphologically distinct vesicle may well be one of the last events reflecting induction of the otic placode, and as such may be the end product of a number of distinct inductive events. For this reason, it has proved difficult to assess the relative contributions of different candidate-inducing tissues (such as the hindbrain, paraxial and axial mesoderm) to the induction of the otic placode (see, for example, Detwiler and Van Dyke, 1950; Fritzsch et al., 1998; Jacobson, 1963a;b;c; Torres and Giráldez, 1998; Waddington, 1937; Yntema, 1950, 1955; Zwilling, 1940). Moreover, in the absence of early markers for the otic placode, it has proved difficult to determine when the initial inductive events commence, and when they have neared completion.

There is thus a clear need for early molecular markers to study otic placode induction. In the present study, we have 
identified four genes (Pax-2, Sox-3, BMP-7 and Notch) that are expressed very early in the developing chick otic placode. We have used these molecular markers, together with classical grafting experiments, to analyze the properties of the responding ectoderm in otic placode induction. We have determined which regions of embryonic ectoderm are competent to give rise to the otic placode, and describe for how long this competence persists. We have also for the first time identified the times at which presumptive otic ectoderm becomes specified and then committed to form the otic placode. Our results provide the first molecular evidence that the induction of the inner ear occurs in multiple stages, some of which can be experimentally uncoupled. In addition, we have begun to map the regions of the embryo that have otic placode-inducing activity, and have determined how long this inducing activity persists in the embryo. This is the first detailed molecular account of the early stages of otic placode induction and serves as a prerequisite to determining the nature of the inducing tissues, and the identity of the molecular signals responsible for the induction of the inner ear.

\section{MATERIALS AND METHODS}

\section{Quail-chick grafts}

Fertilized chick and quail eggs were obtained from a local commercial supplier (AA Labs, Westminster, CA, USA) and incubated at $37.8^{\circ} \mathrm{C}$ in an humidified atmosphere. For this study, embryos were staged according the number of somite pairs formed. Donor and host embryos were visualized by cutting a window in the shell and injecting India ink diluted 1:10 with Ringer's solution under the blastoderm. The vitelline membrane of host and donor embryos was reflected over the region of the surgery using a 30-gauge hypodermic needle. The graft site in the host embryo and the region to be grafted from the donor embryo were prepared using a pulled glass needle. The graft was transferred from donor to host with a glass mouth pipette, and the graft positioned with blunted pulled-glass needles. No attempt was made to preserve rostrocaudal or mediolateral orientation of the grafts, although all grafts were positioned in the correct insideoutside orientation. After grafting, the host eggs were immediately sealed with Scotch tape and returned to the incubator. Embryos were killed after 20-48 hours of incubation, and fixed overnight at $4^{\circ} \mathrm{C}$ in formol saline. Typically, over $90 \%$ of embryos survived the grafting procedure after 24 hours.

\section{Isolation of epiblast tissue}

Epiblast was prepared from Hamburger and Hamilton (HH) stage $3^{+}-$ 4 quail and chick embryos as follows. Yolks were freed of adhering thick albumen and placed in Pannett and Compton saline (Selleck, 1996). The blastoderm was isolated by cutting circumferentially slightly above the equator of the yolk, peeling the vitelline membrane from the yolk with forceps, and peeling the blastoderm off the vitelline membrane. Blastoderms were pooled in Ringer's solution containing $5 \mathrm{mM}$ EDTA to enable easier dissection. An area comprising the anterior $25 \%$ of the region between Hensen's node and the anterior extent of the zona pellucida (see Fig. 2A) was isolated with 30-gauge hypodermic needles, and the epiblast freed from underlying tissue. Epiblasts were stored in Ringer's solution on ice until required (typically 30 minutes to 4 hours), and then cut into appropriately sized pieces immediately prior to grafting.

\section{Collagen gel cultures}

Ectoderm explants for culture were isolated as follows. Embryos were explanted and treated with $1 \mathrm{mg} / \mathrm{ml}$ dispase (Boehringer Mannheim) in Ringer's solution for 15 minutes on ice, and then for 5-15 minutes at $37^{\circ} \mathrm{C}$, depending on the age of the embryo. The digestion was then stopped by washing with complete medium (Minimal Essential Medium (MEM) containing 10\% horse serum and 15\% chick embryo extract) for at least 10 minutes. Ectoderm was dissected free of underlying tissue using 30-gauge hypodermic needles in Ringer's solution, and stored on ice in complete medium. The embryos used in this study were of a sufficiently young age for contaminating tissues to be easily removed. Tissues were washed in DMEM-BS medium (a modification of the chemically defined medium of Bottenstein and Sato, 1979; Wolswijk and Noble, 1989) before being cast in collagen gels. Collagen gels were prepared as previously described (Selleck and Bronner-Fraser, 1995). Briefly, 9 parts collagen solution were combined with 1 part $10 \times$ MEM (Gibco) and brought to neutral $\mathrm{pH}$ with a few drops of $7.5 \%$ sodium bicarbonate. 5-8 explants were suspended in $150 \mu \mathrm{l}$ of gel at widely separated locations, then the gel was allowed to set, and the cultures grown in DMEM-BS at $37^{\circ} \mathrm{C}$ and $5 \% \mathrm{CO}_{2}$ for 24 hours. Cultures were fixed in $4 \%$ paraformaldehyde for 2 hours, and the explants dissected free of the gel prior to performing in situ hybridization.

\section{Immunocytochemistry}

Fixed embryos were equilibrated in PBS containing 15\% sucrose and embedded in $7.5 \%$ gelatin (300 bloom, Sigma) and 15\% sucrose as previously described (Sechrist and Marcelle, 1996). $16 \mu \mathrm{m}$ sections were collected on Superfrost Plus slides (Fisher) and stored at $-20^{\circ} \mathrm{C}$. Sections were blocked in PBS containing $0.1 \%$ Triton X-100 and 5\% foetal bovine serum. Primary antibodies were diluted in blocking solution and applied overnight at $4^{\circ} \mathrm{C}$. Secondary antibodies were diluted in blocking solution and applied for 1 hour at room temperature. Slides were washed in PBS containing $0.1 \%$ Triton X100 several times after each application of antibody, and were washed in distilled water before being mounted in Fluoromount-G (Southern Biotechnology).

The following antibodies were used in this study. A rabbit polyclonal antibody to Pax-2 (Zymed) was used (1:200 dilution); QCPN IgG1 monoclonal antibody (DSHB, University of Iowa, USA) (1:1); a IgG2a monoclonal antibody (TuJ1; Covance) ascites fluid to Beta-III-tubulin (1:1000). Secondary fluorescent and horseradish peroxidase (HRP)-conjugated antibodies were purchased from Jackson Immunochemicals or Southern Biotechnology.

Tissue sections processed with secondary antibodies conjugated to HRP were developed by incubating in $0.1 \mathrm{M}$ Tris, $\mathrm{pH} 7.4$, containing $0.1 \mathrm{mg} / \mathrm{ml}$ diaminobenzidine and $0.003 \%$ hydrogen peroxide, and were washed repeatedly in tap water when the required degree of staining was reached. Sections were scored by counting the number of QCPN+ cells that also expressed one of four molecular markers.

\section{In situ hybridization}

Whole-mount in situ hybridization of embryos and ectoderm explants was performed (with minor modifications) as described in Myat et al. (1996). In situ hybridization on tissue sections, followed by QCPN immunocytochemistry, was performed with modifications as described in Charrier et al. (1999) and Baker and Bronner-Fraser (2000). Detailed protocols are available from the corresponding author on request.

The probes used in this study were obtained from the following sources: chick Pax-2 (provided by Domingos Henrique), chick BMP7 (provided by Brian Huston), chick Sox-3 (provided by Hisato Kondoh), and chick Notch-1 (provided by Julian Lewis and David Ish-Horowicz).

\section{Definitions}

The following terms will be used repeatedly in this paper and are defined here for convenience and clarity. A cell or tissue is competent with respect to a particular developmental fate if it can adopt that fate in an appropriate environment, regardless of whether it normally adopts such a fate in vivo. As a tissue begins to receive inducing 
signals, it is said to be specified with respect to a particular developmental fate if it adopts that fate in a neutral environment, free of any further inducing signals. Specified tissue may, however, adopt alternative fates when challenged with alternative environments, either in culture, or by transplantation to different locations in vivo. A cell or tissue is committed with respect to a particular developmental fate if it recapitulates that fate regardless of its environment. It should be noted that commitment is difficult to definitively demonstrate experimentally, as one can never be sure that the tissue in question has been challenged with all possible alternative environments. It should also be stressed that these definitions are, by nature, operational ones, and at present very little is known regarding the molecular nature of these cell states in any example of embryonic induction (although see Streit et al., 1997, for one such example; Streit and Stern, 1997).

\section{RESULTS}

\section{Molecular markers of the developing otic placode}

We identified four genes that showed early expression in the presumptive chick otic placode (Fig. 1). The paired homeodomain gene Pax-2 was the first gene to be expressed in the presumptive otic placode, at the $4-5$ somite stage (4-5ss). Pax-2 expression assayed by in situ hybridization appeared as a diffuse patch of staining in the ectoderm, several cell diameter lengths lateral to the hindbrain. Over the next few hours, the expression became more intense and encompassed a patch of ectoderm extending medially to the border of the hindbrain, caudally to the border of the first somite and rostrally to the border of rhombomere $1 / 2$ (r1/2). Immunostaining for $\mathrm{Pax}$-2 protein with a polyclonal antiserum revealed an identical spatial and temporal pattern as Pax-2 mRNA expression (Fig. 1C). At the 6ss, Sox-3, an Sry-related homeobox gene was expressed in presumptive placodal ectoderm, followed at the 7ss by BMP-7, a member of the bone morphogenetic protein family. Finally, expression of the transmembrane receptor Notch was seen at the 9-10ss, a few hours before the otic placode invaginates and appears morphologically distinct. Transverse sections of hybridized embryos showed expression of all four genes to be restricted to the ectoderm at the level of the otic placode, with no expression in the underlying mesoderm or endoderm (Fig. 1B), although both Sox-3 and Notch had additional realms of expression in the hindbrain. In the early stages of placode invagination (10-15ss), expression of all four genes was seen in ectoderm immediately surrounding the invaginating placode, but in each case expression became restricted to the otic vesicle as invagination drew to a close. We were not able to tell whether this represented a downregulation of expression, or whether these cells incorporated into the otocyst during invagination.

\section{Competence of embryonic ectoderm to form the otic placode}

We next sought to determine what regions of the embryo were competent to give rise to the otic placode. Different populations of embryonic quail ectoderm were grafted in place of presumptive otic ectoderm in chick hosts (Figs 2 and 3). Embryos were allowed to survive for 24-48 hours and assayed for expression of the molecular markers described in the preceding section, together with immunochemistry for the quail-specific marker QCPN. The ages of host embryos receiving grafts varied between 3 ss and $10 \mathrm{ss}$, and we found no evidence to suggest that the age of the host within this age range affected the experimental outcome (data not shown).

We performed a preliminary series of control experiments to ensure that the act of grafting did not itself seriously disrupt otic placode development. Presumptive otic ectoderm from quail embryos was grafted isotopically in place of presumptive otic ectoderm in chicks. In 15 out of 18 operations, quail ectoderm incorporated normally into the host, expressed Pax2 protein and contributed to an invaginated otic placode or vesicle (Fig. 2B). In the remaining three operations, the grafted ectoderm contributed to Pax-2-positive ectoderm immediately rostral or caudal to the otic vesicle (not shown).

To test the competence of ectoderm to form the otic placode, we first grafted ectoderm from the most anterior region of epiblast of $\mathrm{HH}$ stage $3^{+}-4$ quail embryos in place of presumptive otic ectoderm in chick hosts (Fig. 2A). This region of epiblast does not normally form the otic placode (GarciaMartinez et al., 1993; Rosenquist, 1966), contributing instead mainly to extra-embryonic ectoderm. However, $88 \%$ of operated embryos contained quail ectoderm expressing Pax-2 protein, while in $53 \%$ of embryos, the $\mathrm{Pax}-2^{+}$quail tissue contributed to an invaginated otic placode or vesicle (Fig. 2C; $n=17)$. To confirm that the grafts were contributing to otic placode tissue, a number of operated embryos were processed for in situ hybridization using probes for BMP-7 $(n=17)$, Sox-3 $(n=15)$ and Notch $(n=16)$, together with QCPN immunocytochemistry. $88 \%, 87 \%$ and $80 \%$ of operated embryos contained quail cells in or adjacent to the otic vesicle that expressed BMP-7, Sox-3 or Notch, respectively. (Fig. 2D). Thus competence to form an otic placode is present in embryonic ectoderm as early as $\mathrm{HH}$ stage 3-4.

As the otic placode continues to develop, it begins to express regional markers of differentiation, corresponding to three main classes of cells in the developing inner ear: sensory, nonsensory and neuronal. To determine if anterior epiblast could be induced to express a regional marker of ear differentiation, 10 chick embryos receiving grafts of $\mathrm{HH}$ stage $3^{+}-4$ quail anterior epiblast were allowed to develop for 48 hours and labeled with TuJ1, a pan-neuronal BIII-tubulin antibody as a marker for differentiating neurons. Quail cells labeled with the TuJ1 antibody were seen delaminating from the otic vesicle in 9/10 embryos examined (Fig. 2E), demonstrating that anterior epiblast is competent to generate at least one differentiated cell type from the otic vesicle.

To determine how the competence of a given region of donor ectoderm varied with age, we grafted ectoderm from different stages of quail embryos into chick hosts. Quail ectoderm was taken from either the level of the midbrain $(n=70)$, which will normally form the ophthalmic division of the trigeminal placode (Baker et al., 1999; Stark et al., 1997), or from the trunk between the level of the presumptive 5th to 8 th somites ( $n=44$; Fig. 3A). Both populations of ectoderm were competent to generate $\mathrm{Pax}-2^{+}$cells, and in many cases these cells contributed to the invaginating otic placode (Fig. 3C,D). However, the competence of both midbrain and trunk-level ectoderm declined rapidly with age, such that by the 10 ss, neither population was competent to express Pax-2 or contribute to the invaginating placode (Fig. 3B,E). In these 
grafts of older ectoderm, the Pax-2+ placode was split by a region of Pax-2-quail tissue (Fig. 3E). By the 6ss, no grafts of either population of ectoderm formed a $P a x-2^{+}$vesicle, although some grafts still expressed Pax-2 without invaginating (data not shown). A similar pattern of diminishing competence with age was seen in a small number of embryos analyzed with the other molecular markers BMP-7, Sox-3 and Notch (not shown).

\section{Specification of an otic placode fate in embryonic ectoderm}

We next examined when presumptive otic placode tissue becomes specified to express markers of the otic placode, by culturing fragments of placodal ectoderm from different ages of chick embryos in isolation in collagen gels (Fig. 4A). In order to reduce the possibility of the culture conditions affecting the differentiation of the explanted tissue, we used a chemically defined medium (Bottenstein and Sato, 1979; Wolswijk and Noble, 1989) containing no serum or embryo extract, with only insulin present to assist tissue survival. Cultures were examined after 24 hours for expression of Pax-2 $(n=101)$ and BMP-7 ( $n=177)$. Significant numbers of explants $(61 \%)$ were specified with respect to Pax-2 expression at the 5-6ss, while significant numbers of explants $(53 \%)$ were specified with respect to BMP-7 expression at the 7-8ss (Fig. 4C). In both cases, specification appeared to occur at the approximate time that both genes are expressed in vivo (Fig. 1A). In all cases, expression of either Pax- 2 or $B M P-7$ was restricted to a small part of the explanted ectoderm. Examples of explants are shown in Fig. 4B. Similar results were seen with smaller numbers of explants examined with Sox-3 and Notch, and with explants maintained for 48-60 hours (not shown).

\section{Commitment of embryonic ectoderm to an otic placode fate}

We examined when presumptive placodal ectoderm becomes committed to express Pax-2 by grafting presumptive otic ectoderm from quail embryos of different ages into the lateral trunk of chick hosts (n=46; Fig. 5A). Grafts of young presumptive otic placodal ectoderm (3-4ss) did not express Pax-2 when grafted into the lateral trunk (Fig. 5B,C). However, beginning at the 56 ss, quail ectoderm from the otic placode region expressed $P a x-2$ in the trunk. In some cases, the Pax-2 $2^{+}$ ectoderm formed a distinct epithelial vesicle; in other cases, the $P a x-2^{+}$ ectoderm did not invaginate. By the $9-10$ ss, nearly $100 \%$ of quail grafts formed Pax $-2^{+}$epithelial vesicles in the lateral trunk (Fig. 5B,C). Interestingly, the levels of Pax-2 protein expression seen in the lateral trunk were often lower than normal, although obviously above background, suggesting that signals in the cranial region may be necessary for the maintenance as well as the induction of this gene, or alternatively that signals in the lateral plate mesoderm may partially downregulate $\operatorname{Pax}-2$ in these grafts.

\section{Location of Pax-2-inducing activity in the embryo}

The preceding experiments demonstrate that ectoderm from a variety of locations in the early embryo is competent to express Pax-2 and invaginate to form an otic vesicle. However, only a restricted region of embryonic ectoderm goes on to become specified and finally committed to forming the inner ear. We therefore sought to map the
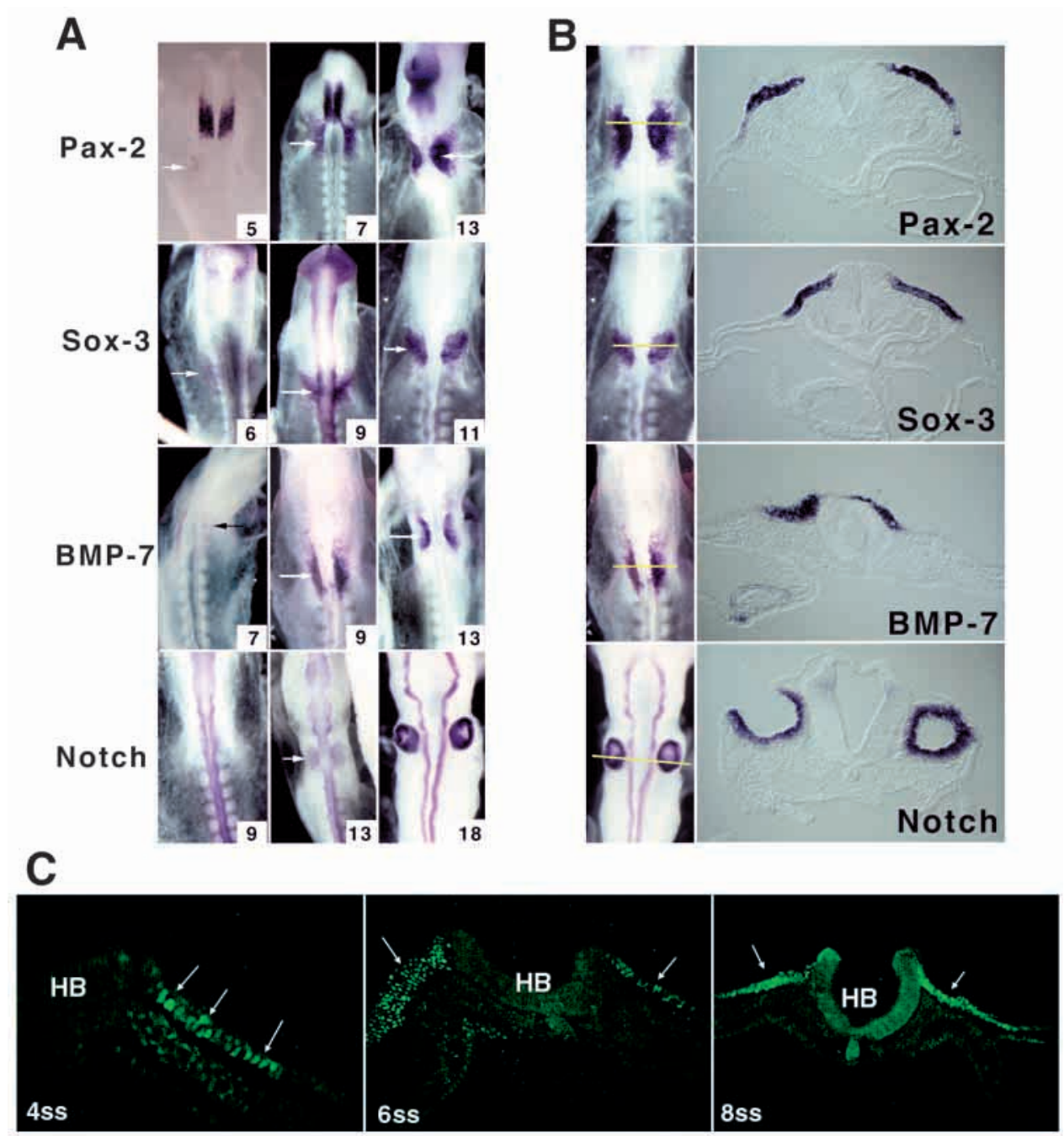

Fig. 1. Expression of four molecular markers for the chick otic placode. (A) Whole-mount in situ hybridization of Pax-2, Sox-3, BMP-7 and Notch. The numbers refer to the age of the embryo expressed as number of somite pairs (ss). The white arrows indicate the otic placode. (B) Sections through embryos processed for whole-mount in situ hybridization. The approximate plane of section is indicated by the yellow lines. Note the staining is exclusively ectodermal.

(C) Immunostaining of 4ss (detail), 6ss and 8ss chick embryos with an anti-Pax-2 antiserum. Pax-2 expression is indicated with arrows. 
location of Pax-2-inducing activity in the developing embryo. Anterior epiblast from $\mathrm{HH}$ stage $3^{+}-4$ quail embryos was grafted to different locations along the rostrocaudal axis of the embryo ( $n=63$; Fig. 6A). Embryos were allowed to survive for 24 hours, sectioned and examined for Pax-2 expression and the presence of quail tissue with the QCPN antibody. The results are presented graphically in Fig. 6B.

Quail anterior epiblast grafted in place of midbrain-level ectoderm never expressed Pax-2 (although some grafts expressed the ophthalmic trigeminalspecific marker Pax-3; Stark et al., 1997; Baker et al., 1999; not shown), and never invaginated to form a vesicle $(n=15)$. A small number of quail grafts to the trunk also failed to express Pax2 or to invaginate to form a vesicle $(0 / 4)$. 5 out of 6 grafts to the presumptive nodose placode adjacent to the first two somites expressed Pax-2, but did not form an epithelial vesicle. Quail epiblast was also grafted inside the neural tube at two levels; the hindbrain $(n=6)$, and the neural tube at the level of somites 5-10 $(n=6)$. In neither case did the quail tissue express Pax-2, although the tissue appeared to survive and often incorporated into the host neural tube.

As mentioned above, quail anterior epiblast grafted to the level of the presumptive otic placode was readily induced to express Pax-2 (88\% of grafts; $n=17)$ and $53 \%$ of cases also invaginated to form a vesicle. When anterior epiblast was grafted to the level of rhombomeres $1-3$, a smaller percentage of embryos contained $\mathrm{Pax}-2^{+}$quail tissue $(44 \%$; $n=9)$. In general, these Pax $-2^{+}$grafts lay quite close to the host otic vesicle; grafts to $1-3$ that came to reside more rostrally never expressed Pax-2, although some did invaginate to form a Pax-2-vesicle. Thus, the region of the embryo in which anterior epiblast tissue was capable of expressing Pax-2 was tightly restricted to the axial level of the hindbrain.

\section{Duration of inducing activity in region of the otic placode}

The preceding work demonstrated a Pax-2-inducing activity at the hindbrain level of the embryo. Epiblast grafted at this level also invaginated to form an epithelial vesicle. In order to determine for how long such activities are present in the embryo, and whether the two activities are experimentally separable, quail anterior epiblast was grafted to the otic placode region of older chick (11-22ss) hosts and processed for Pax-2 and QCPN immunocytochemistry (Fig. 7A).

As described above, quail anterior epiblast grafted to the
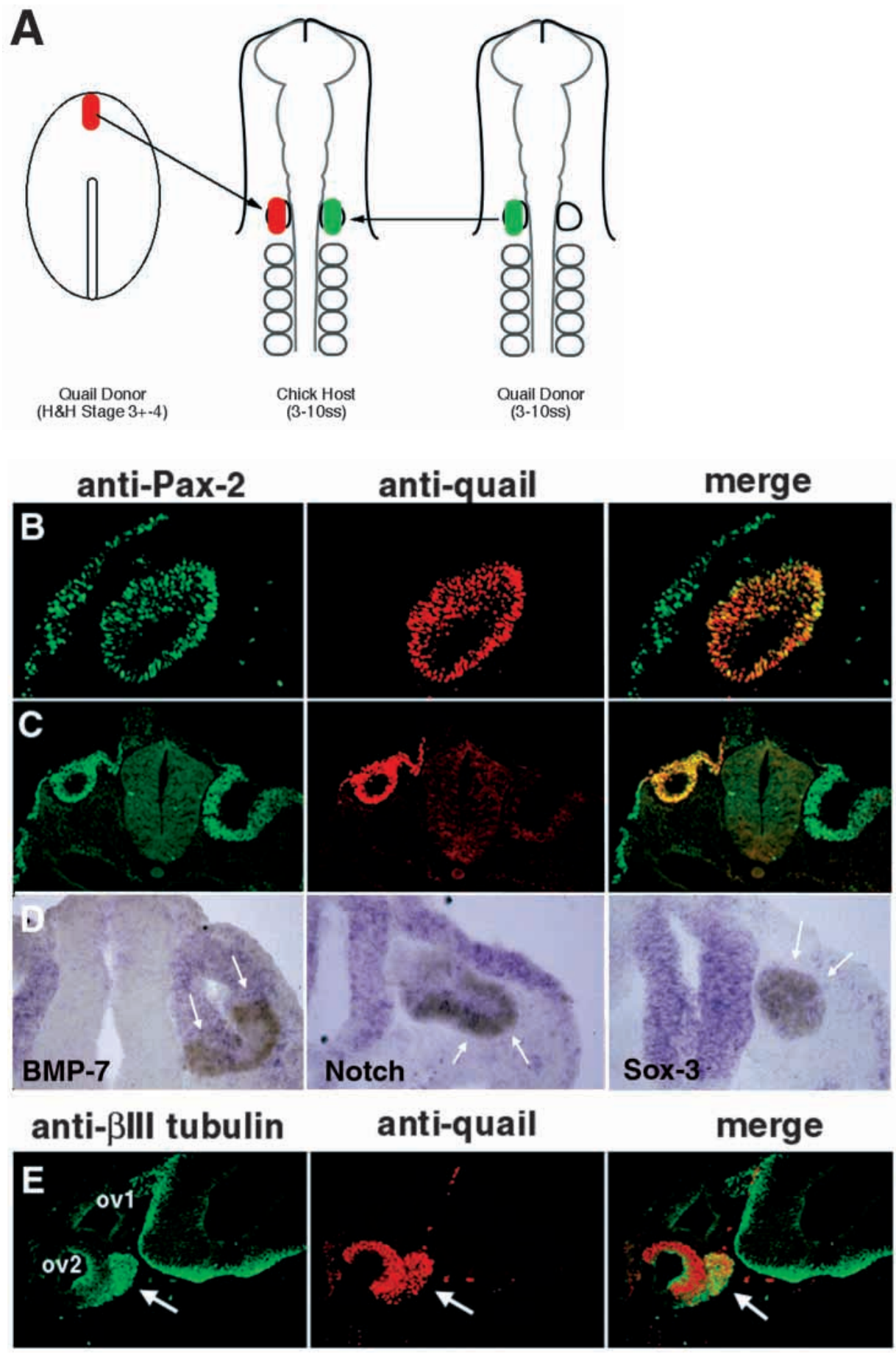

Fig. 2. (A) Chick embryos received grafts of quail anterior epiblast from stage 3-4 embryos or isotopic grafts of quail placodal ectoderm. (B) An isotopic quail graft gives rise to a Pax-2+, quail-derived otic vesicle. (C) Quail anterior epiblast is also competent to give rise to a Pax-2+ otic vesicle, although the quail vesicle is smaller than the contralateral host vesicle.

(D) Anterior epiblast grafts also express BMP-7, Notch and Sox-3. Quail cells, in brown, are indicated with arrows. (E) Grafts of anterior epiblast also give rise to TuJ1+ neurons after 48 hours survival. The delaminating, quail-derived ganglion is shown with an arrow. Note the quail tissue forms a second otic vesicle (ov2) below a host otic vesicle (ov1). For details, see text. 


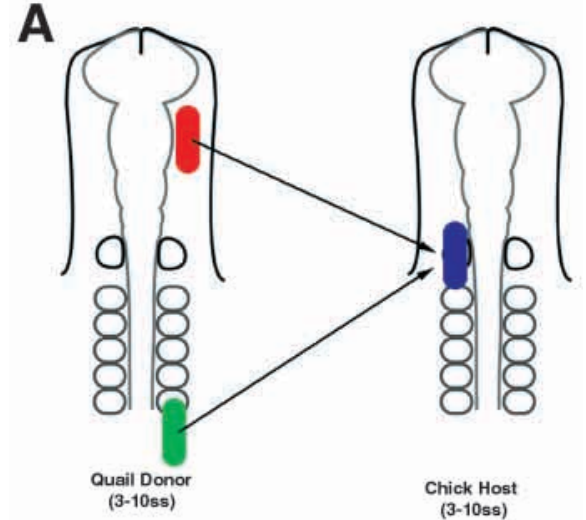

Fig. 3. Competence of trunk and midbrain level ectoderm to contribute to the otic placode. (A) Trunk or midbrain level ectoderm was grafted to the presumptive otic placode region. (B) Graph of Pax-2 expression in trunk or midbrain ectoderm grafts of different ages. The number of grafts from either region expressing Pax-2 declines rapidly with age. Figures above the bars refer to the number of grafts analyzed. (C) Example of young (3ss) quail midbrain level ectoderm grafted to the presumptive otic placode region. (D) Example of young (3ss) quail trunk level ectoderm grafted to the presumptive otic placode region. Note the graft forms an ectopic vesicle next to the host otic vesicle. (E) Graft of older (9ss) trunk ectoderm no longer expresses Pax-2 (arrowheads), although the host ectoderm on either side of the graft continues to express Pax-2 (arrows), suggesting that the host placodal field is split by the graft. For details, see text.

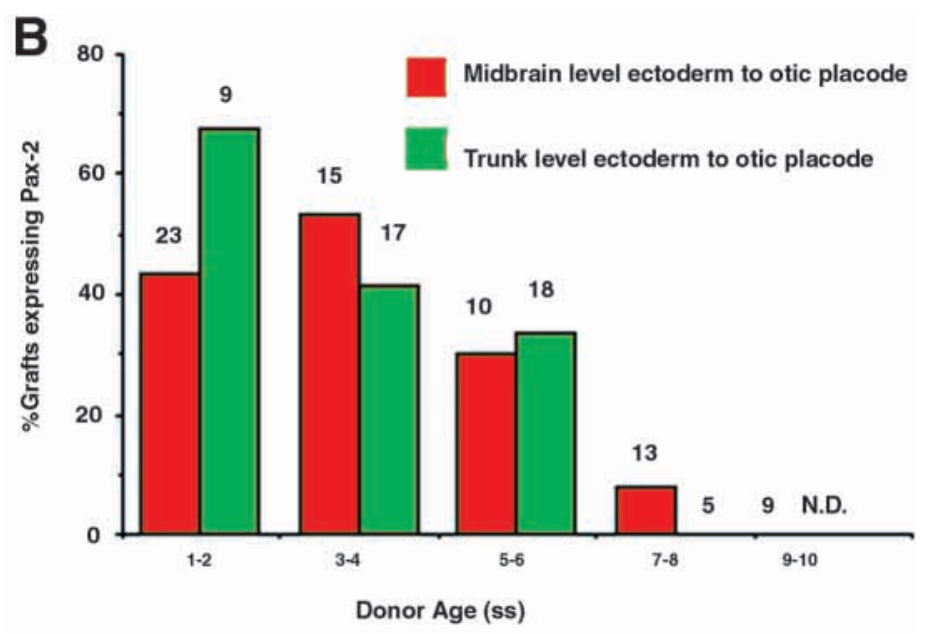

anti-Pax-2

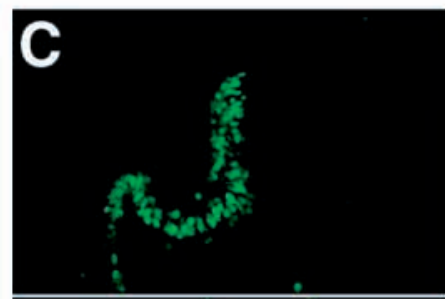

anti-quail

merge
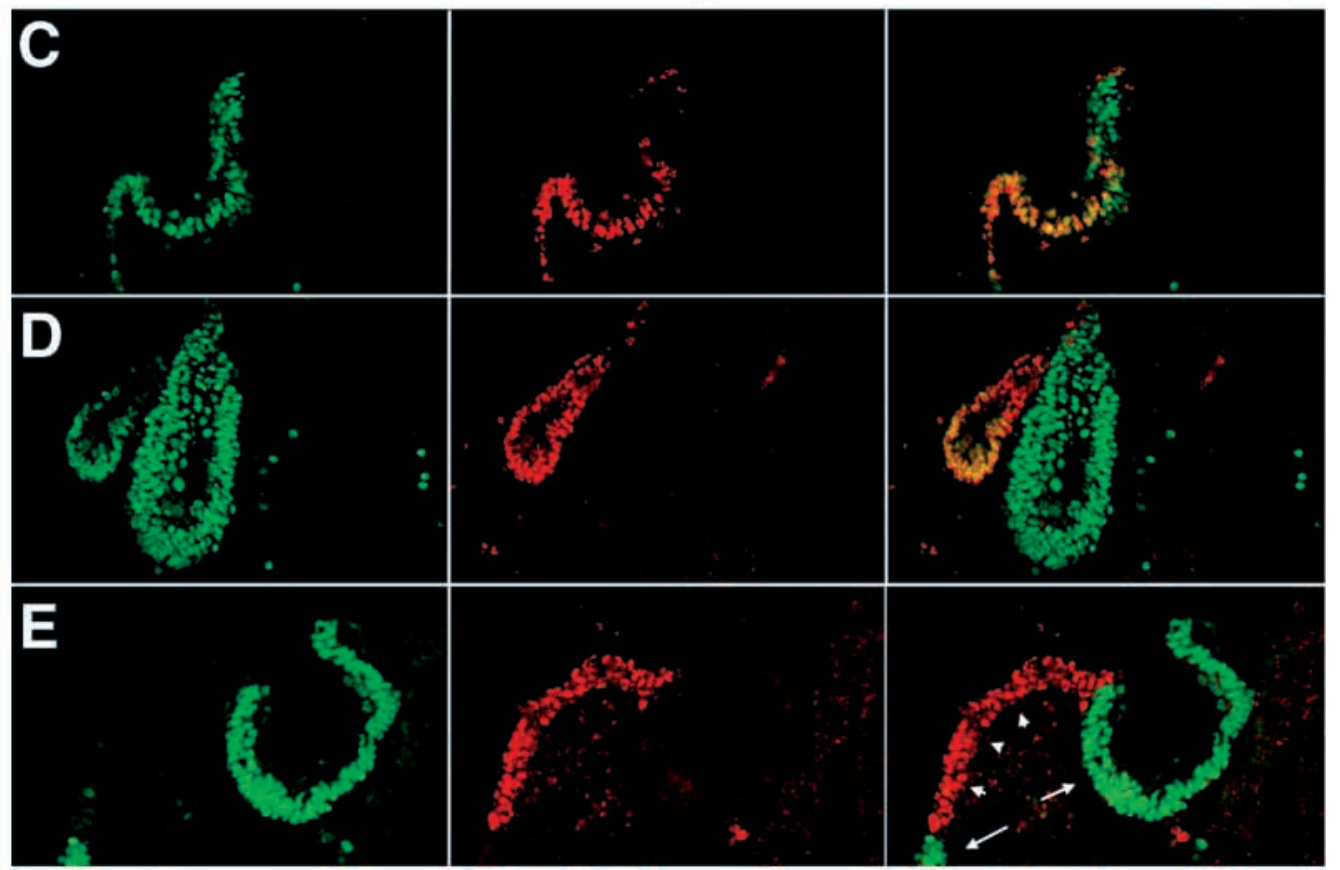

presumptive otic placode of hosts aged between 3 and 10ss readily expressed $\mathrm{Pax}-2$ and frequently invaginated to form a vesicle. When the same population of epiblast was grafted to older hosts (11-21ss), however, a different result was seen (Fig. 7B). In most cases the epiblast invaginated to form an epithelial vesicle $(91 \% ; n=23)$, but the number of embryos in which the invaginated quail tissue expressed $P a x-2$ was greatly reduced (22\%). To determine whether the Pax-2 negative vesicles seen in these older embryos still expressed other markers of the otic placode, a number of embryos were processed for in situ hybridization for BMP-7, Sox-3 or Notch (Fig. 7C). Once more, most grafts formed epithelial vesicles (49/54). However, a few grafts expressed Notch (4/18), while 14/18 grafts expressed BMP-7 and 15/18 expressed Sox-3. In summary, when anterior epiblast is grafted into progressively older hosts, it becomes less likely to express Pax-2 and Notch, more likely to form an epithelial vesicle, and remains equally likely to express $B M P$ -
7 and Sox-3. These experiments show that different aspects of otic placode differentiation can be experimentally uncoupled from one another.

\section{DISCUSSION}

\section{Molecular markers of the developing otic placode}

Despite eight decades of study, the cellular and molecular processes leading to the formation of the inner ear remain elusive. One reason for this lack of progress has been summarized succinctly by Gurdon. In concluding his review of 1987 he wrote: "I believe that the greatest obstacle to the molecular analysis of induction over many decades may have been the imprecision and late appearance of the assays used, which often depend on morphological assessment many days after the inductive response has started". Any attempt to 

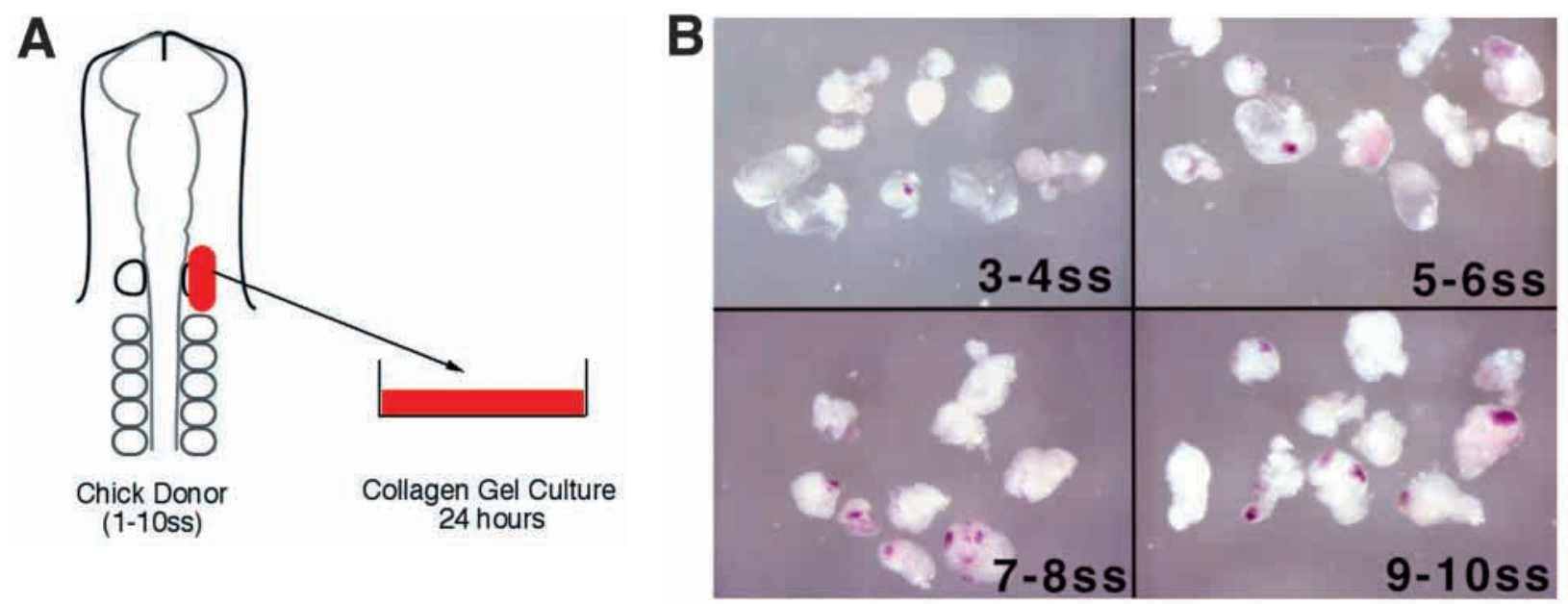

Fig. 4. Specification of presumptive placodal ectoderm. (A) Presumptive otic ectoderm of different ages was explanted in collagen gels and cultured in a defined medium for 24 hours. (B) Examples of explant cultures, processed for in situ hybridization with Pax-2. Increasing numbers of explants express Pax-2 with age. (C) Graph showing the percentage of explants expressing either $B M P-7$ or Pax-2 with age. Figures above the bars refer to the number of explants analyzed. For details, see text.

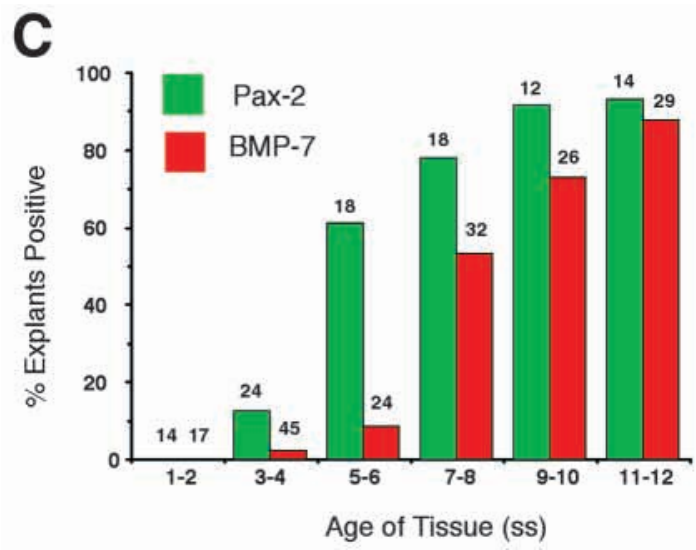

understand the events leading to inner ear induction must therefore make use of early and specific markers of the otic placode.

In the present study we have described four genes that are expressed very early in the induction of the chick otic placode. All four genes are first expressed before the otic placode is morphologically identifiable as an invaginating pit adjacent to the hindbrain. The earliest gene, Pax-2, is expressed in presumptive placodal ectoderm at the $4-5$ ss, whilst Notch, the last gene to be expressed, is detected shortly before the invagination of the placode at the 12-14ss. This expression data suggests that induction of the otic placode in birds is probably occurring significantly earlier than previously thought. Moreover, evidence from fish, amphibians and mice suggests that other genes (such as Pax-8, Dlx-5 and Dlx-3) may be expressed before Pax-2 in the developing otic placode (Acampora et al., 1999; Depew et al., 1999; Ekker et al., 1992; Heller and Brandli, 1999; Pfeffer et al., 1998). If this is also shown to be true in birds, it raises the possibility that otic placode induction may begin as early as the end of gastrulation. In this regard, we note that two studies considering the roles of FGF-3 (Represa et al., 1991) and the neural crest (Meier, 1978a,b) in otic placode induction were actually examining embryos well after the initial inductive events had likely occurred. Our present results suggest that future studies of otic placode induction in birds might profitably focus on the gastrulating and early postgastrulation embryo.
It is important to note that none of the four molecular markers used in this study are completely specific for the otic placode. For example, Pax-2 is expressed in the midbrainhindbrain junction, and also much later in the epibranchial placodes of the chick (Baker and Bronner-Fraser, 2000), whilst Notch is expressed throughout the developing central nervous system of the embryo. However, the coexpression in ectoderm of these four genes is truly specific for the otic placode. In the present study, the majority of our quail-chick grafting experiments were only analyzed with antibodies to Pax-2 and a quail-specific marker, QCPN, for convenience. In order to be confident that Pax-2 immunoreactivity was indicative of otic placodal ectoderm, some samples were analyzed by hybridizing embryos with probes for BMP-7, Sox-3 or Notch, together with QCPN immunocytochemistry. Similar percentages of embryos were found to express Pax-2, Sox-3, BMP-7 or Notch in invaginating ectoderm.

In addition to their utility as molecular markers of the early inner ear, the four genes in this study are likely to serve important functions in the formation of this organ. For example, mice homozygous for a mutation of the Pax-2 gene have severe inner ear defects, lacking both the entire cochlea, and a large part of the VIIIth cranial ganglion (Torres et al., 1996). It remains to be determined whether mutations in the other genes reported here have similarly profound effects on inner ear development. 


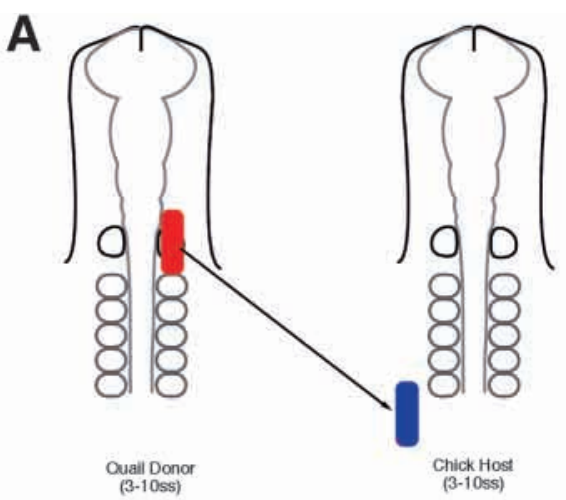

(3-10ss) $\underset{\text { (3.10ss) }}{\text { Chick Host }}$

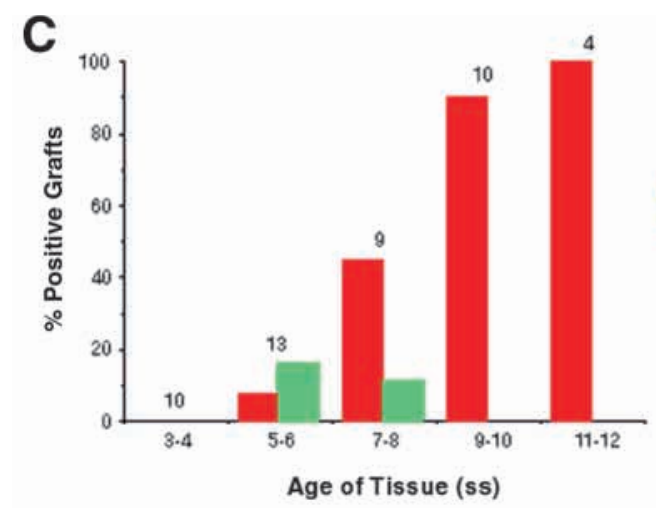

anti-quail

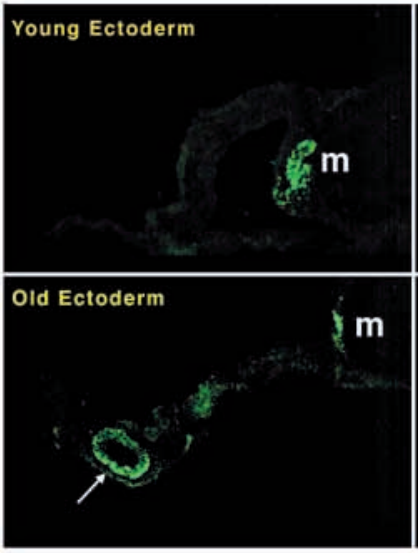

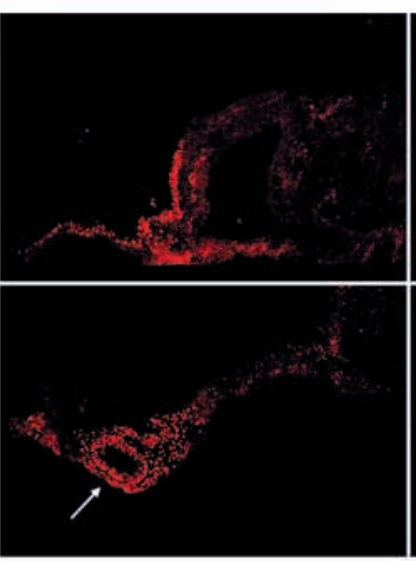

merge

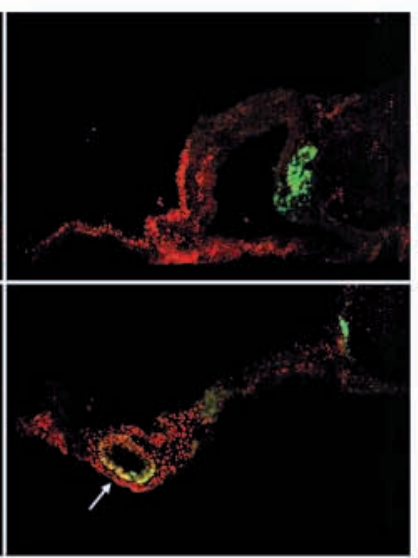

$\%$ Pax-2+ grafts forming vesicle

$\%$ Pax-2+ grafts not forming vesicle

Fig. 5. Commitment of placodal ectoderm. (A) Quail otic ectoderm of different ages was grafted to the lateral trunk. After 24 hours, embryos were examined for Pax-2 expression and QCPN staining. (B) Young ectoderm (from a 4ss donor) does not express Pax-2 in the lateral trunk, whereas older ectoderm (from a 10ss donor) not only expresses Pax-2, but also forms an epithelial vesicle (arrows). The Pax-2+ mesonephros is indicated (m). (C) Graph showing presumptive placodal ectoderm becomes increasingly committed with age. Note that some grafts of intermediate age can express Pax-2, but do not form epithelial vesicles. Figures above the bars refer to the number of grafts analyzed. For details, see text.

\section{Competence to form the otic placode}

Previous work in chicken and amphibian embryos suggests that many regions of the early embryo are competent to form an otic placode if grafted to an appropriate location (for example, Kaan, 1926; Yntema, 1950; Jacobsen, 1963a; Gallagher et al., 1996). Our experiments using molecular markers of the developing otic placode have confirmed these observations. We find that anterior epiblast taken from embryos as young as stage $3^{+}$is highly competent to form an otic placode. Competence to form an otic placode thus exists in a population of ectoderm not normally fated to give rise to the embryo proper from an early stage of embryonic development. We do not yet know whether competence of ectoderm to form the otic placode is contingent on extracellular signals, as has been reported for neural induction in birds (Streit et al., 1997; Streit and Stern, 1997). A previous study in Xenopus showed that anterior ventral ectoderm is initially only poorly competent to form the otic placode, and that this competence increases, and then declines again with age (Gallagher et al., 1996). The earliest anterior epiblast taken in our study (at stage $3^{+}$) is already highly competent to form the otic placode; we have not tested whether earlier chick epiblast is more or less competent to form the placode.

In our experiments, we find that epiblast tissue not only contributes to the otic placode but it can also go on to produce one differentiated derivative of the otic placode, $\mathrm{TuJ} 1^{+}$neurons. We cannot be certain at present that such neurons are true VIIIth ganglion neurons, as specific markers for this neuronal population are not available. Given that the neurons in our grafts are clearly delaminating from a graft-derived otic vesicle, however, it seems likely that they are indeed VIIIth ganglion neurons.

Our results with molecular markers confirm previous morphological observations that ear-forming competence of ectoderm located outside the presumptive otic placode declines precipitously with age. Ectoderm from the midbrain level and from the rostral regions of the trunk is competent to form an otic placode at young ages (1-4ss), but this competence is completely lost by the 10-12ss. Interestingly, it appears that competence to form an invaginated epithelial vesicle is lost before the competence to express one of the four markers of the otic placode. This ability to experimentally uncouple different aspects of otic placode induction is discussed further below. A recent study from our laboratory (Baker et al., 1999) showed that cranial ectoderm was competent to express Pax-3 in the ophthalmic division of the trigeminal placode, whereas trunk ectoderm of a comparable age was not. In contrast, our present results show that rostral trunk ectoderm is competent to form the otic placode for a limited period of time. Vogel and Davies (1993) have shown that trunk ectoderm can also contribute to the nodose placode, thus a given population of ectoderm cells of a given age can be competent to contribute to one type of cranial placode, but not to another. The reasons for such differential responsiveness will not become clear until we have a molecular understanding of competence, rather than simply an operational one.

\section{The time frame of otic placode induction}

The present study has for the first time charted the time over 
Fig. 6. Location of Pax-2-inducing activity in the embryo. (A) Quail anterior epiblast was grafted to seven locations in chick hosts. After 24 hours, embryos were examined for the expression of Pax-2 in the graft tissue. (B) Graph showing the induction of $\mathrm{Pax}-2$ at the different locations. Induction is only observed at rostrocaudal levels between the first two somites and rhombomeres 2-3. Figures above the bars refer to the number of grafts analyzed.
A

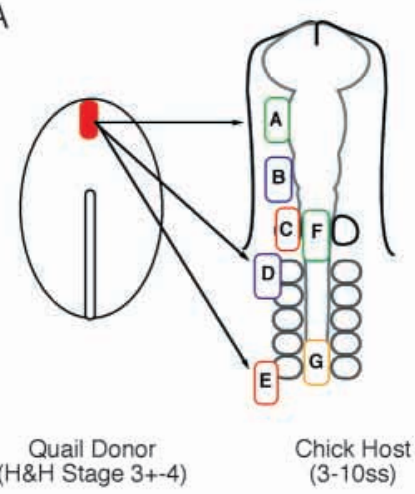

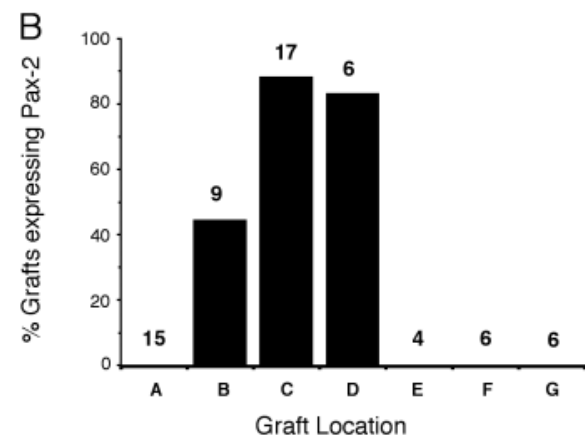

which otic placode induction occurs in birds. We determined when presumptive otic ectoderm becomes specified by culturing ectoderm in collagen gels in the absence of inducing factors. We find that significant specification of the otic placode with respect to Pax-2 and BMP-7 occurs at almost the same time that these genes are expressed in vivo, at the $5 \mathrm{ss}$ and 7 ss, respectively. A similar temporal relationship between specification and in vivo gene expression has been reported for Pax-3 in the trigeminal placode (Baker et al., 1999). Moreover, we observed that expression of BMP-7 and Pax-2 was maintained in explants cultured for 48-60 hours (data not shown), suggesting that once expression of these two genes is initiated in placodal ectoderm, no further signals are required from surrounding tissues to maintain gene expression.

Commitment of ectoderm to an otic placode fate was tested by grafting presumptive placodal ectoderm to the lateral trunk. We chose this site as being likely to have no otic placodeinducing activity, although we cannot say this definitively. We found that in a relatively short space of time after placode specification (by 9-10ss), the otic ectoderm is committed to express Pax-2 and to form an epithelial vesicle. Thus, on the basis of our assays, cranial ectoderm is committed to forming the inner ear before the otic placode becomes morphologically visible as an invaginating pit. Our results using molecular markers are in good agreement with Waddington's early results in the chick (Waddington, 1937). He found that presumptive otic ectoderm grafted into the amniocardiac vesicle before the 9ss rarely gave rise to morphologically identifiable ears, whereas grafts taken after this age gave rise to small ear-like structures in the majority of cases. A similar pattern was seen in grafts of presumptive otic tissue to the midbrain level or limb bud (Herbrand et al., 1998). Ginsburg (1995) examined otic placode commitment in three urodele and seven anuran species by grafting presumptive otic ectoderm to the belly of host embryos. Interestingly, she observed that the time at which ectoderm becomes committed to an otic placode fate varies dramatically between species, occurring at the yolk plug stage in some species and as late as the neural tube stage in others. The reason for this interspecies heterochrony is unknown, although Ginsburg suggests this may be due to the fact that gastrulation occurs progressively later in the course of amphibian evolution, thus linking placode induction with events occurring during or after gastrulation.

This is the first instance in which the specification and commitment of the otic placode has been mapped with molecular probes. The importance of carefully mapping the time course of otic placode induction in this way is shown by a previous study, which examined the role of FGF-3 in otic
A

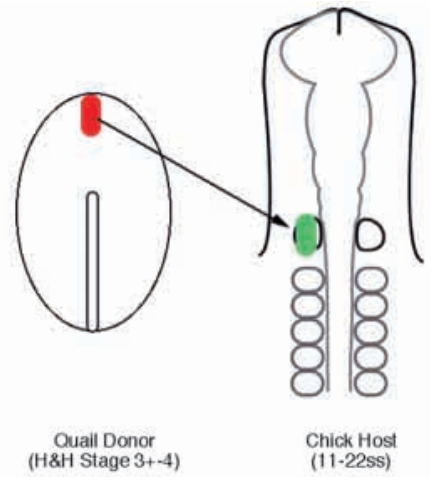

Fig. 7. Differential regulation of otic placode markers in older hosts. (A) Quail anterior epiblast was grafted to the presumptive otic placode region of 1122ss chick hosts. (B) Although the quail tissue forms an epithelial vesicle, it frequently does not express Pax-2 in older hosts. Note Pax-2 expression in adjacent ectoderm and in the hindbrain (arrows). Quail cells also express BMP-7 and Sox-3, but very little Notch mRNA. The QCPN+ quail cells are stained brown. Note that Notch expression occurs either side of the quail graft (arrows), but very little Notch expression is seen in the graft (arrowheads).

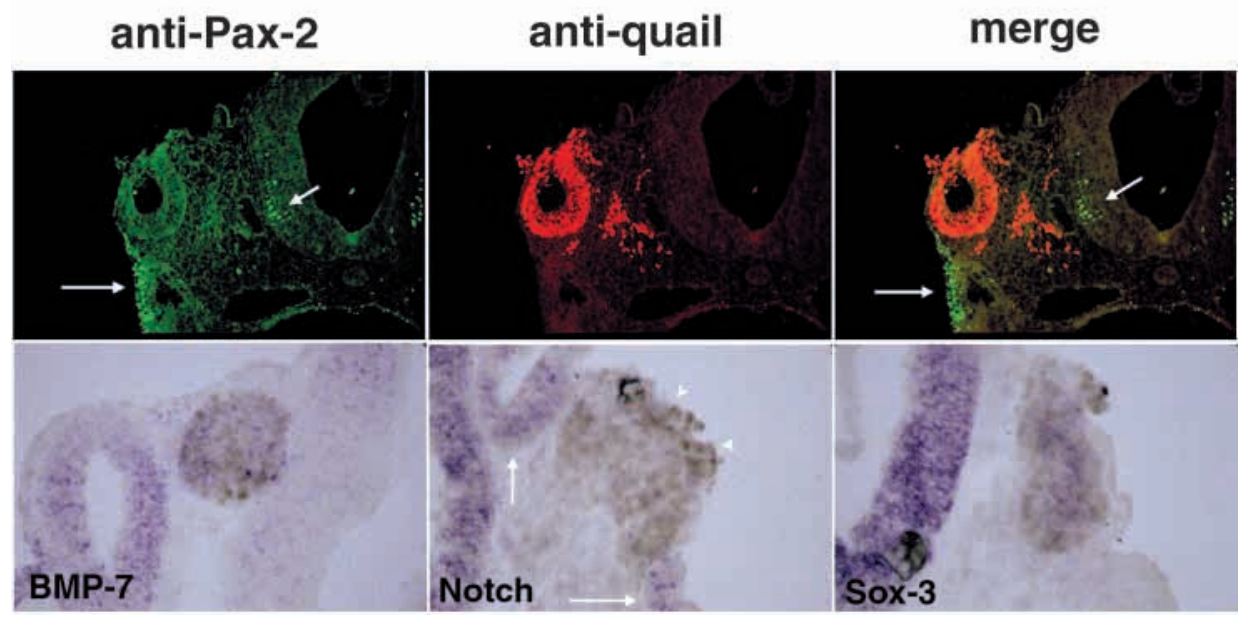


placode induction and invagination (Represa et al., 1991). This study used embryos from the 9-10ss, whereas our present data suggest that this ectoderm was already committed to an otic placode fate.

\section{Spatial and temporal distribution of the inducing activity}

We have mapped the spatial distribution of Pax-2-inducing activity in vivo by grafting competent epiblast from quail embryos to different positions along the rostrocaudal axis of chick hosts. We find that a region extending from the first two pairs of somites forward to rhombomeres 2 and 3 has the ability to induce Pax-2 protein. A slightly more anterior region of the embryo can also induce the formation of epithelial vesicle-like structures. We do not yet know which tissues are responsible for the inducing activity, although we are currently investigating this question. Our present results are in marked contrast to a recent study of the ophthalmic trigeminal placode from our laboratory (Baker et al., 1999). This showed that a Pax-3inducing activity could be localized to virtually the entire extent of the neural tube, but that spatial and temporal changes in competence meant that only a restricted region of ectoderm along the rostrocaudal axis was able to respond to this activity. In contrast our present results show that much of the ectoderm along the rostrocaudal axis is competent to express Pax-2, but that Pax-2-inducing activity is either confined to a much more narrow region, or else is present over a wide area but repressed anterior to $r 2 / 3$ and posterior to the first few somites. This emphasizes once more that induction of different ectodermal placodes is likely to occur by different mechanisms.

When we grafted quail anterior epiblast to the otic placode region of older chick hosts (between 11-21ss), we noticed several changes in the level of inducing activity in comparison with younger hosts (3-10ss). The number of grafts expressing Pax -2 and Sox-3 dropped sharply, whereas the number of grafts forming an epithelial vesicle increased, with BMP-7 and Notch expression remaining essentially unchanged. This suggests that different markers of otic placode differentiation can be regulated independently from one another, and that signals inducing or maintaining expression of these markers exert their effects over different time courses.

\section{Otic placode induction proceeds by multiple, experimentally separable steps}

An important conclusion to come from our study is that the induction of the otic placode does not occur in a single unitary decision, but rather as a series of steps that are likely to be independently regulated, and which can be experimentally uncoupled from one another. First, our commitment experiments show that at early stages, presumptive placodal ectoderm grafted to the lateral trunk is committed to express Pax-2, but not to form an otic vesicle (Fig. 5). Second, the competence of midbrain- or trunk-level ectoderm to form an otic vesicle is lost more rapidly than the competence of this ectoderm to express Pax-2. Third, grafts of anterior epiblast to old (11-21ss) hosts show that competent epiblast can be induced to form an epithelial vesicle independently of the expression of Pax-2, and Notch, but not independently of BMP7 or Sox-3 (Fig. 7). Fourth, we find that grafts of anterior epiblast to the level of r1-3 often form an epithelial vesicle, but do not express Pax-2.
These results reinforce those of Ginsburg, originally carried out in the 1930s but not published in the West until 1995 (Ginsburg, 1995). In her studies of several amphibian species, Ginsburg demonstrated that presumptive otic ectoderm became committed to form an otic vesicle before being committed to form sensory epithelium when transplanted to the ventral body wall. In another study, Noden and Van de Water (1986) report that presumptive otic placode ectoderm grafted to more anterior locations can form an epithelial vesicle bearing sensory cells, but cannot generate neurons. When grafted over trunk somites, placodal ectoderm forms an epithelial vesicle, but not neurons or sensory cells (Noden and Van de Water, 1986). Finally, Bober and colleagues showed that the regulation of Pax-2 and $N k x 5.1$ can be regulated independently from one another in the invaginated chick otocyst (Herbrand et al., 1998). Taken together, these findings suggest a model of inner ear development in which successive waves of inducing signals cause the progressive differentiation of placodal tissue into a mature sensory organ. Our results using molecular markers show that different steps in the differentiation of the otic placode can be regulated independently at the molecular as well as the morphological level. At present, however, the nature and identity of the factors that regulate these steps is not known. A recent study (Vendrell et al., 2000) has suggested that FGF-3 may play a role in otic placode induction, although it is still not clear whether this molecule is either necessary or sufficient for placode induction, nor whether it acts directly on competent ectoderm, or indirectly by up-regulating other inducing molecules.

\section{Conclusion}

As a first step to understanding the molecular basis for the induction and patterning of the otic placode, we have used a panel of molecular markers to determine the time at which the otic placode is specified and then committed. The use of molecular, rather than morphological markers reveals induction of the chick otic placode to occur earlier than had been previously thought. In addition, we now show that different aspects of placode induction can be regulated independently from one another. The molecular basis of the extracellular and intracellular pathways that govern induction of the otic placode are still not clear. However, we believe that the use of early molecular markers will allow us to determine the identity of the tissues, signaling molecules and transcription factors that act together to induce and pattern the inner ear.

We thank Julian Lewis, David Ish-Horowicz, Domingos Henrique, Brian Huston and Hisato Kondoh for cDNA probes. A.K.G. wishes to thank Clare Baker for her support and advice, and for her comments on the manuscript, Heather Etchevers for advice on the paraffin in situ protocol and Butch Welch for assistance with color image processing. A.K.G. is supported by the House Ear Institute and an NIH Small Project Grant, DC03630-01. This work was also supported by NIH grants NS 13815 and NS 36585 to M.B.-F.

\section{REFERENCES}

Acampora, D., Merlo, G. R., Paleari, L., Zerega, B., Postiglione, M. P., Mantero, S., Bober, E., Barbieri, O., Simeone, A. and Levi, G. (1999). Craniofacial, vestibular and bone defects in mice lacking the Distal- lessrelated gene Dlx5. Development 126, 3795-3809. 
Baker, C. V., Stark, M. R., Marcelle, C. and Bronner-Fraser, M. (1999). Competence, specification and induction of Pax-3 in the trigeminal placode. Development 126, 147-156.

Baker, C. V. and Bronner-Fraser, M. (2000). Establishing neuronal identity in vertebrate neurogenic placodes. Development, in press.

Bottenstein, J. E. and Sato, G. H. (1979). Growth of a rat neuroblastoma cell line in serum-free supplemented medium. Proc. Natl. Acad. Sci. USA 76, 514-517.

Charrier, J. B., Teillet, M. A., Lapointe, F. and Le Douarin, N. M. (1999). Defining subregions of Hensen's node essential for caudalward movement, midline development and cell survival. Development 126, 4771-4783.

Depew, M. J., Liu, J. K., Long, J. E., Presley, R., Meneses, J. J., Pedersen, R. A. and Rubenstein, J. L. (1999). Dlx5 regulates regional development of the branchial arches and sensory capsules. Development 126, 3831-3846.

Detwiler, S. R. and Van Dyke, R. H. (1950). The role of the medulla in the differentiation of the otic vesicle. J. Exp. Zool. 113, 179-199.

Ekker, M., Akimenko, M. A., Bremiller, R. and Westerfield, M. (1992). Regional expression of three homeobox transcripts in the inner ear of zebrafish embryos. Neuron $\mathbf{9}, 27-35$.

Fritzsch, B., Barald, K. F. and Lomax, M. I. (1998). Early embryology of the vertebrate ear. In Development of the Auditory System, vol. 9 (ed. E. W. Rubel, A. N. Popper and R. R. Fay), pp. 80-145. New York: Springer.

Gallagher, B. C., Henry, J. J. and Grainger, R. M. (1996). Inductive processes leading to inner ear formation during Xenopus development. Dev. Biol. 175, 95-107.

Garcia-Martinez, V., Alvarez, I. S. and Schoenwolf, G. C. (1993) Locations of the ectodermal and nonectodermal subdivisions of the epiblast at stages 3 and 4 of avian gastrulation and neurulation. J. Exp. Zool. 267, 431-446.

Ginsburg, A. S. (1995). Determination of the labyrinth in different amphibian species and its correlation with determination of the other ectoderm derivatives. Roux's Arch. Dev. Biol. 204, 351-358.

Grainger, R. M. (1992). Embryonic lens induction: shedding light on vertebrate tissue determination. Trends Genet. 8, 349-355.

Grainger, R. M. (1996). New perspectives on embryonic lens induction. Semin. Cell. Dev. Biol. 7, 149-155.

Groves, A. K. and Anderson, D. J. (1996). Role of environmental signals and transcriptional regulators in neural crest development. Dev. Genet. 18, 6472.

Groves, A. K., George, K. M., Tissier-Seta, J. P., Engel, J. D., Brunet, J. F. and Anderson, D. J. (1995). Differential regulation of transcription factor gene expression and phenotypic markers in developing sympathetic neurons. Development 121, 887-901.

Gurdon, J. B. (1987). Embryonic induction - molecular prospects. Development 99, 285-306.

Hamburger, V. and Hamilton, H. L. (1951). A series of normal stages in the development of the chick embryo. J. Morphol. 88, 49-92.

Heller, N. and Brandli, A. W. (1999). Xenopus Pax-2/5/8 orthologues: novel insights into Pax gene evolution and identification of Pax-8 as the earliest marker for otic and pronephric cell lineages. Dev. Genet. 24, 208-219.

Herbrand, H., Guthrie, S., Hadrys, T., Hoffmann, S., Arnold, H.-H., Rinkwitz-Brandt, S. and Bober, E. (1998). Two regulatory genes, cNkx5-1 and cPax2 show different responses to local signals during otic placode and vesicle formation in the chick embryo. Development 125, 645654.

Jacobson, A. G. (1963a). The determination and positioning of the nose, lens and ear. I. Interactions within the ectoderm, and between the ectoderm and underlying tissues. J. Exp. Zool. 154, 273-283.

Jacobson, A. G. (1963b). The determination and positioning of the nose, lens and ear. II. The role of the endoderm. J. Exp. Zool. 154, 285-291.

Jacobson, A. G. (1963c). The determination and positioning of the nose, lens and ear. III. Effects of reversing the antero-posterior axis of epidermis, neural plate and neural fold. J. Exp. Zool. 154, 293-303.
Jacobson, A. G. (1966). Inductive processes in embryonic development. Science 152, 25-34.

Jacobson, A. G. and Sater, A. K. (1988). Features of embryonic induction. Development 104, 341-359.

Kaan, H. W. (1926). Experiments on the development of the ear of Amblystoma punctatum. J. Exp. Zool. 46, 13-61.

Meier, S. (1978a). Development of the embryonic chick otic placode. I. Light microscopic analysis. Anat. Rec. 191, 447-458.

Meier, S. (1978b). Development of the embryonic chick otic placode. II. Electron microscopic analysis. Anat. Rec. 191, 459-477.

Myat, A., Henrique, D., Ish-Horowicz, D. and Lewis, J. (1996). A chick homologue of Serrate and its relationship with Notch and Delta homologues during central neurogenesis. Dev. Biol. 174, 233-247.

Noden, D. M. and Van de Water, T. R. (1986). The developing ear: Tissue origins and interactions. In The Biology of Change in Otolaryngology (edited), pp. 15-46. Elsevier.

Pfeffer, P. L., Gerster, T., Lun, K., Brand, M. and Busslinger, M. (1998). Characterization of three novel members of the zebrafish Pax2/5/8 family: dependency of Pax5 and Pax8 expression on the Pax2.1 (noi) function. Development 125, 3063-3074.

Represa, J., Leon, Y., Miner, C. and Giraldez, F. (1991). The int-2 protooncogene is responsible for induction of the inner ear. Nature 353, 561-563.

Rosenquist, G. C. (1966). A radioautographic study of labeled grafts in the chick blastoderm. Contributions to Embryology, Carnegie Institute of Washington 38, 73-110.

Sechrist, J. and Marcelle, C. (1996). Cell division and differentiation in avian embryos: techniques for study of early neurogenesis and myogenesis. Methods Cell Biol. 51, 301-329.

Selleck, M. A. J. (1996). Culture and microsurgical manipulation of the early avian embryo. Methods Cell Biol. 51, 1-21.

Selleck, M. A. J. and Bronner-Fraser, M. (1995). Origins of the avian neural crest: the role of neural plate-epidermal interactions. Development 121, 525538.

Stark, M. R., Sechrist, J., Bronner-Fraser, M. and Marcelle, C. (1997). Neural tube/ectoderm interactions are required for trigeminal placode formation. Development 124, 4287-4295.

Streit, A., Sockanathan, S., Perez, L., Rex, M., Scotting, P. J., Sharpe, P. T., Lovell-Badge, R. and Stern, C. D. (1997). Preventing the loss of competence for neural induction: HGF/SF, L5 and Sox-2. Development 124, 1191-1202.

Streit, A. C. and Stern, C. D. (1997). Competence for neural induction: HGF/SF, HGF1/MSP and the c-Met receptor. CIBA Found. Symp. 212, 155165.

Torres, M. and Giráldez, F. (1998). The development of the vertebrate inner ear. Mech. Dev. 71, 5-21.

Torres, M., Gomez-Pardo, E. and Gruss, P. (1996). Pax-2 contributes to inner ear patterning and optic nerve trajectory. Development 122, 33813391.

Vendrell, V., Carnicero, E., Giraldez, F., Alonso, M.T. and Schimmang, T. (2000). Induction of inner ear fate by FGF3. Development 127, 2011-2019.

Vogel, K. S. and Davies, A. M. (1993). Heterotopic transplantation of presumptive placodal ectoderm changes the fate of sensory neuron precursors. Development 119, 263-276.

Waddington, C. H. (1937). The determination of the auditory placode in the chick. J. Exp. Biol. 14, 232-239.

Wolswijk, G. and Noble, M. (1989). Identification of an adult-specific glial progenitor cell. Development 105, 387-400.

Yntema, C. L. (1950). An analysis of induction of the ear from foreign ectoderm in the salamander embryo. J. Exp. Zool. 113, 211-244.

Yntema, C. L. (1955). Ear and Nose. In Analysis of Development, (ed. V. Hamburger), pp. 415-428. Philadelphia, PA: Saunders.

Zwilling, E. (1940). The determination of the otic vesicle in Rana pipiens. J. Exp. Zool. 86, 333-343. 